\title{
Polarimetric Temporal Information for Urban Deformation Map Retrieval
}

\author{
Luca Pipia, Xavier Fabregas, Albert Aguasca, Carlos \\ Lopez-Martinez, Jordi. J. Mallorqui, \\ Remote Sensing Lab. (RSLab), Signal Theory and \\ Communications Department (TSC), \\ Universitat Politècnica de Catalunya (UPC), Barcelona, Spain
}

\author{
Oscar Moraline \\ Institut Cartogràfic de Catalunya (ICC), \\ Barcelona, Spain
}

\begin{abstract}
In this work, a preliminary study on the use of polarimetric persistent scatterers for differential interferometric applications within an urban environmental is presented. The PoISAR measurements campaign that the RSLab of UPC is carrying on in the village of Sallent using an X-Band groundbased SAR sensor is first described. The work is then focused on the additional information the knowledge of full scattering matrix $[S]$ may provide with respect to the single polarization approach. The problem of instability of the polarimetric signature of urban targets is also analyzed. Finally, a solution based on the search of repeated patterns in the long-temporal profile of pixels' polarimetric signature is briefly introduced.
\end{abstract}

\section{INTRODUCTION}

The great challenge of the remote sensing SAR community in the next years is to explore the possibility to exploit the high amount of information contained in the polarimetric SAR data to improve the performance of already assessed singlepolarization techniques. Owing to its remarkable achievements, the differential interferometry is surely one of the most representative research areas. The complementary techniques of the Permanent Scatterers (PS)[1] and Coherent Pixels (CPT) [2] have been demonstrated to be extremely successful depending on the features of the environment to be monitored. In both cases, the most critical step is the selection of points to be trusted for the retrieval of an accurate deformation map. The lack of spaceborne polarimetric SAR sensors, and consequently of long temporal PolSAR data, prevented from trying to extend the mathematical formulation of these advanced differential methods to multiple polarization channels. The coherence optimization of pixels along the whole interferograms stack through polarization base transformations, or the combined research of stable pixels among the HH-HV-VV channels, are just examples of subjects never considered before. The Remote Sensing Laboratory (RSLab) of the Universitat Politècnica de Catalunya (UPC) is carrying on a measurements campaign in the village of Sallent [3] using its polarimetric ground-based SAR sensor in collaboration with the Institut Cartogràfic de Catalunya (ICC). The PolSAR data monthly acquired at X-Band since July 2006 for the monitoring of a mining induced subsidence phenomenon give for the first time the opportunity to perform

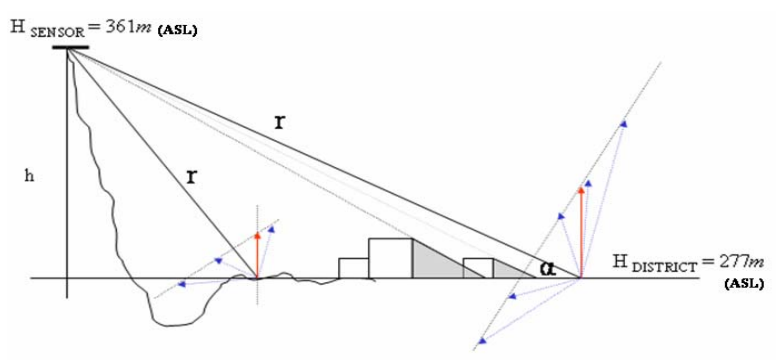

Fig.1: GBSAR acquisition geometry.

this type of analysis. The daily and long-temporal behavior of the polarimetric response of deterministic targets selected within the observed district will be here analyzed. The problem of temporal stability of amplitude and phase parameters will be interpreted as geometrical modifications of the targets themselves due to the normal human activity. A criterion for the selection of reliable pixels based not on the dispersion index $D_{A}[1]$ but on the regularity of both amplitude and phase profiles will be introduced. The quantitative comparison between the polarimetric and single-polarization selection of stable pixels, and during the diurnal and nocturnal monitoring activity, will be finally carried out.

\section{MEASURING ACTIVITY DESCRIPTION}

When a ground-based SAR solution is adopted for the monitoring activity of an area, a critical issue is the sensor location: the higher the observation angle with respect to the horizontal plane, the higher the opportunity to reduce the shadowing effects due to closer targets. The observation geometry of the campaign in Sallent is sketched in Fig.1. In order to solve zero-baseline errors and to guarantee the placement of the linear unit exactly at the same position, a concrete basement with an iron screws grid was constructed. In each day of measurements, the sensor acquired data every 20 minutes for at least 7 hours. An aperture of 2 meters with a sampling step of $1 \mathrm{~cm}$ was employed. Despite the deployment high precision assured by the screw grid structure, a sub-pixel spatial correlation technique was used to assess that no further coregistration step was needed. The same strategy was employed to estimate and to compensate the miscorregistration among the polarimetric channels related to the length of cables and antennas layout. In Table 1 it is given 


\begin{tabular}{|c|c|c|c|c|c|c|c|c|}
\hline $\begin{array}{c}29 / 06 / 06 \\
\text { DAY1 }\end{array}$ & $\begin{array}{c}26 / 07 / 06 \\
\text { DAY2 }\end{array}$ & $\begin{array}{c}19 / 09 / 06 \\
\text { DAY3 }\end{array}$ & $\begin{array}{c}20 / 10 / 06 \\
\text { DAY4 }\end{array}$ & $\begin{array}{c}14 / 11 / 06 \\
\text { DAY5 }\end{array}$ & $\begin{array}{c}28-29 / 11 / 06 \\
\text { DAY6 }\end{array}$ & $\begin{array}{c}18-19 / 12 / 06 \\
\text { DAY7 }\end{array}$ & $\begin{array}{c}13-14 / 02 / 07 \\
\text { DAY8 }\end{array}$ & $\begin{array}{c}14-15 / 03 / 07 \\
\text { DAY9 }\end{array}$ \\
\hline $11: 00 / 8: 00$ & $7: 00 / 13: 00$ & $10: 20 / 16: 30$ & $9: 00 / 15: 00$ & $11: 30 / 17: 30$ & $17: 30 / 8: 30$ & $17: 00 / 9: 00$ & $18: 00 / 8: 30$ & $17: 00 / 9: 00$ \\
\hline
\end{tabular}

Table 1: Acquisition Schedule

a schedule of the days when acquisitions were gathered. It can be noticed that in the first 5 days a diurnal monitoring was performed, whereas for the last 4 days nightly ones were chosen.

The analysis of a trihedral response showed a polarization purity of around $30 \mathrm{~dB}$ between the co-polar and cross-polar channels. This allowed to describe the polarimetric distortion of the system by a diagonal matrix and to apply the calibration method proposed in [4]. Two reference point scatterers, a pure co-polar (Trihedral) and a pure cross-polar calibrator (Bruderhedral $45^{\circ}$ degree tilted respect to the line of sight), were hence used for the purpose.

\section{ONE-DAY POLARIMETRIC BEHAVIOUR}

The deterministic nature of the targets within the urban environment suggests the use of a coherent technique for their polarimetric analysis. In Fig. 2 the RGB image obtained with the three components of the Pauli's Decomposition applied to a calibrated dataset is displayed. A brief analysis points out the main presence of just one dominant scattering mechanism in most of pixels within the urban area. The red pixels indicate a single-bounce (trihedral-like) behavior whereas the green ones denotes a double-bounce (dihedral-like) scattering. Yellow pixels, which denote the simultaneous presence of both single and double bounces, are detectable too, although less frequently. Blue points are related to a pure cross-polar scattering generated by multiple-reflections phenomena. As it can be noticed, this Pauli's component is almost absent within the scenario. The unique bright blue scatterer in the near range corresponds to the Bruderhedral used for the cross-talk calibration. The use of the span parameters to modulate the brightness of the RGB image allows to focus the attention on the brightest pixels that are likely to be most important scatterers.

Although an urban area is mainly made up of high reflectivity targets, the stability of the amplitude and phase parameters turned out to be extremely dependent on the part of the day data were acquired. Atmospheric phase artifacts related to the refractive index fluctuation during a whole day had to be first removed [5]. The propagation properties are independent of the polarization of the electromagnetic wave but, in order to perform a study of the phase stability along a whole day, these effects must be taken into account. The plots of Fig. $3 \mathrm{a}$ and $3 \mathrm{~b}$ describe the polarimetric signature of the same high reflectivity pixel about $400 \mathrm{~m}$ from the sensor during a nocturnal (a) and diurnal (b) monitoring activity. The night response is very stable for the first 18 acquisitions (from 6 to 11 p.m.), then a jump affects both amplitude and phase quantities introducing a new but still stable polarimetric behavior. The last part of the profile, corresponding to the

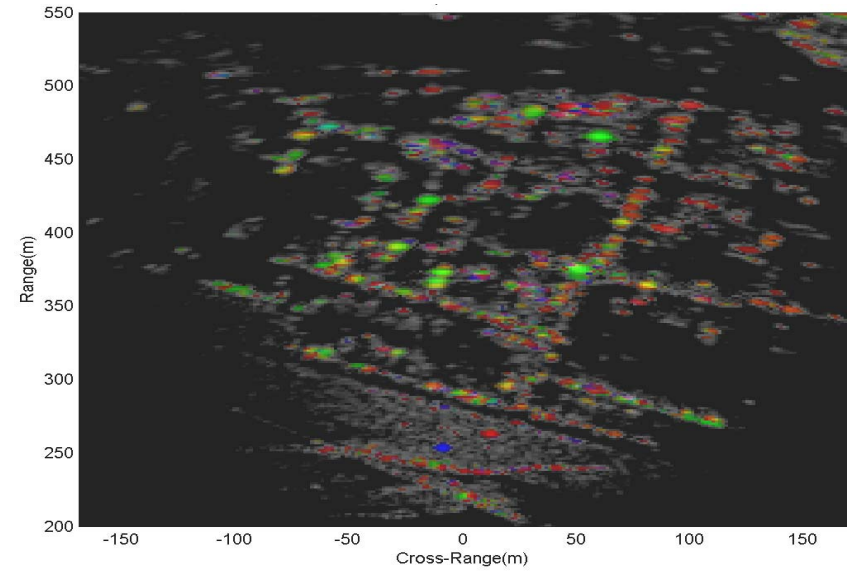

Fig2: Pauli's RGB Image weighted by span of the Sallent's district.
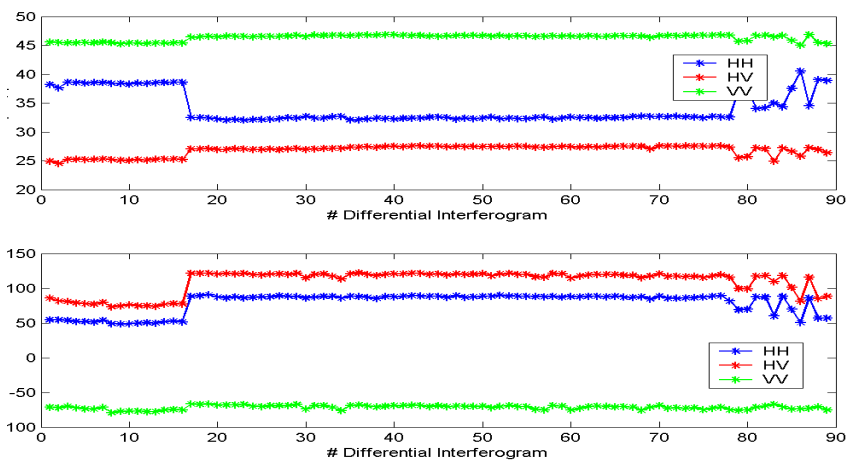

(a) Nocturnal Monitoring from $5 \mathrm{pm}$ on Dec. $18^{\text {th }}$ to $9 \mathrm{am}$ on Dec. $19^{\text {th }} 2006$.


(b) Diurnal Monitoring from 9am to $15 \mathrm{am}$ on Oct. $20^{\text {th }} 2006$.

Figs. 3: Amplitude [dB] and Phase [Deg] temporal evolution of the same high reflectivity pixel during the night (a) and during the day(b).

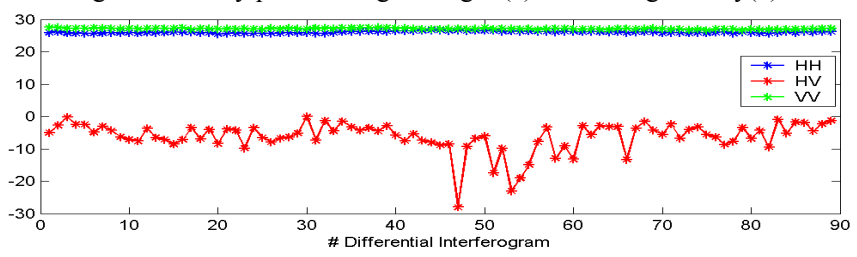

Fig. 4: Amplitude $[\mathrm{dB}]$ profile of a corner reflector from $5 \mathrm{pm}$ on Dec. $18^{\text {th }}$ to 9am on Dec. $19^{\text {th }} 2006$. 



(a)

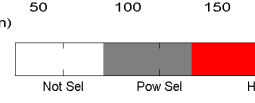

period from the 7a.m. to 9a.m, is characterized by higher
fluctuations. If the diurnal profile (Fig.3b) of the same pixels is now observed, the profile stability decreases even more.

The suspicion of any system failure must be excluded. In both cases, the temporal profile of a corner reflector placed within the scene was studied: a standard deviation of less than $0.5 \mathrm{~dB}$ for amplitude and 1 degree for phase was estimated. Moreover, no jumps in both profiles were detected, exactly as for other profiles corresponding to other targets (Fig.4). Possible residual errors of atmospheric artifacts compensation must be excluded as well, since they cannot justify so sudden and high steps, neither for the phase nor for the amplitude. Besides, they should affect close pixels the same way due to the correlation length of atmospheric phenomena, but this is not observed. For the same reason, any coregistration residual error must be excluded as well.

On the contrary, this instability must be understood in terms of the dynamic configuration that an urban area can assume during a whole day and that directly affects the stability of the targets response at X-Band. The structural properties that this type of environment presents during the diurnal hours are likely to be more mutable than during the nocturnal ones. The highest contribution of back scattered power, as pointed out by the Pauli's image, comes from the double bounce scattering mechanism, that implies and interaction between vertical walls of buildings and the street pavement. For this reason, the observation geometry of a ground-based sensor is definitely supposed to increase but not to generate these effects: they are thought to be related to changes in the location of the different point targets within the complex deterministic environment. Since only the human activities can justify these changes, they are expected to be more probable during the day than the night.

The main objective of the measurements campaign in Sallent is to monitor the subsidence phenomena induced by the mining activity of the past years [3]. The opportunity given by the GB-SAR platform to gather as many datasets as desired can be used to improve the quality of the phase information: a daily temporal filtering at [S] matrix level can be considered for the additive noise reduction without loss of resolution. The deterministic nature of the targets and the fact that they are always observed in the same way allows to describe the scattering matrix $[\mathrm{S}]$ measured at the scanning $i$ as:

$$
[S]_{i}^{\text {meas }}=[S]^{\text {real }}+[n]_{i}
$$

where the $[\mathrm{S}]^{\text {real }}$ depends only on the position of the sensor and of the target, whereas $[n]_{i}$ is a $2 \times 2$ complex matrix that takes into account the additive noise contribution to each polarimetric channel. Since the position of the sensor does not change during a whole day, under the hypothesis that the polarimetric signature of the target keeps constant during the whole day, $[\mathrm{S}]^{\text {real }}$ can be better estimated by applying a temporal coherent average to the $N$ datasets daily acquired by the sensor:

$$
\widehat{S}^{\text {real }}=\frac{1}{N} \sum_{i=1}^{N}[S]_{i}^{\text {meas }}
$$

Atmospheric artefacts must be compensated before using the eq.(2). The instability of the polarimetric signature of the target observed both during the day and the night (Figs.3) prevents from averaging directly the whole set of one-day collection for each pixel. The development of a criterion for the selection of reliable stable pixel within the image becomes essential. The dispersion index $D_{\mathrm{A}}$ defined for the PSs [1] cannot be applied to ground-based acquisitions, or at least it cannot be justified in the same way. In fact, the amplitude samples collected in this case do not describe the statistic of the target RCS, neither Rice nor any other distribution. The observation geometry keeps constant and the resolution of $1 \times 2$ $\mathrm{m}^{2}$ provided by the sensor in the area of the district excludes the presence of a clutter within each cell. Consequently, the backscattering mechanism turns into a deterministic more than a statistical process, whose samples are just corrupted by additive noise.

Nevertheless, the amplitude is a key parameter: high fluctuations or a jumps in the amplitude profile automatically exclude a regularity of the phase behavior. In order to perform a useful selection of reliable pixels, a two steps iterative algorithm has been defined. The first step is seeking out for the amplitude jumps higher than a fixed threshold: the daily profile is splitted into several segments. Each segment is then compared with the not contiguous ones and joined if fulfilling the regularity condition. 
The algorithm stops when the longest stable amplitude profile is obtained. With this solution, also the targets that generally show a constant behavior in the three polarimetric channels apart from few anomalous sub-intervals, maybe due to temporary external factors, are selected. Once the amplitude criterion has been fulfilled, the corresponding phase profile is analyzed. A new jump threshold is introduced and the algorithm is applied again. If the final length of the vector containing the reliable information is longer than two third of the total number of one-day acquisitions, the pixel is chosen, otherwise it is discarded.

The criterion of selection is completely independent of the wave polarization and can be directly extended to all the elements of [S]. An example of the result of the polarimetric selection obtained in case of diurnal and nocturnal acquisitions is compared in Fig.5a and 5b. An amplitude mask has been applied to filter out the weaker scatterers. As already remarked by the Pauli's decomposition, the total backscattered power is mainly distributed into the two copolar channels whereas the information contribution of the cross-polar term is negligible. Consequently, HV turns out to be the less stable channel and provides very few useful pixels. On the contrary, the $\mathrm{HH}$ and VV polarization carry complementary information. Their combined use makes the number of points increase more than $50 \%$ if compared with the selection obtained in the single polarization approach (Table 2). Moreover, the nocturnal monitoring allows to select approximately the double of pixels than in the diurnal acquisitions. This fact confirms that the amplitude and phase instability at X-Band is related to the dynamic geometry the urban targets may assume during a whole day, as a consequence of the human daily activities.

In order to perform a longer temporal analysis, verifying that the signature of the target is exactly the same becomes crucial. An example is given in Fig.6, where the result of the three components of the Pauli's decomposition concerning a very strong scatterer is plotted throughout almost seven months. If in the diurnal measurements the target's signature is very instable and no constant behavior is detectable, a well-defined pattern repeats at approximately the same time (11:30 p.m) every day in the nocturnal ones. The temporal filtering performed for each day of measurements in the first situation would not reduce the additive noise contribution: it would cause just the mixing of different targets behaviors with a consequent loss of information. On the contrary, the [S] time average can be profitably applied to the nocturnal intervals where the geometrical properties of the target do not change. Different averaged polarimetric datasets can be now combined for the extraction of the differential phase since the hypothesis of target stability is fulfilled also for long temporal analysis. This study, described for a single pixel, must be repeated for the rest of the image. The resulting cloud of points may be now treated as the new polarimetric input data required by advanced DInSAR techniques.

\begin{tabular}{|c|c|c|c|c|c|c|c|c|}
\hline \multirow[b]{3}{*}{ Diurnal } & \multirow{2}{*}{\multicolumn{2}{|c|}{$\begin{array}{c}\text { HH } \\
\mathrm{AMP}\end{array}$}} & \multicolumn{2}{|c|}{$\mathbf{V V}$} & \multicolumn{2}{|c|}{ HHUVV } & \multicolumn{2}{|c|}{ HHกVV } \\
\hline & & & AMF & $\mathrm{PH}$ & $\mathrm{AM}$ & $\mathrm{PH}$ & $\mathrm{AM}$ & PH \\
\hline & 4531 & 1241 & 4843 & 1038 & 6997 & 1595 & 2377 & 684 \\
\hline Nocturnal & 5355 & 2291 & 4765 & 1983 & 7469 & 3124 & 2651 & 1150 \\
\hline
\end{tabular}

Tabla 2: Pixels Selection:Diurnal vs Noctural Measurements. Amplitude TH: 2dB, Phase TH: $7^{\circ}$



Fig.6 : Long temporal profile of Pauli's components of a high reflectivity urban target (see Table 1 for the correspondent dates)

\section{CONCLUSION}

In this paper, the monitoring activity that the Remote Sensing Laboratory (RSLab) of UPC is carrying on in the village of Sallent using a polarimetric GB-SAR system has been presented. The problem of high instability of the signature of urban targets due to the dynamic nature of this man-made environment has been pointed out. A criterion of stability adapted to the GB-SAR case has been introduced to show the great potential of polarimetry for the selection of persistent scatterers within an urban area in long temporal analysis. Nocturnal monitoring must be preferred not only for atmosphere behavior but mainly for urban environment higher stability. The next step will be now to combine in a unique formulation the differential information of the whole scattering matrix to look into the improvement that such a solution may bring to the deformation maps retrieval.

\section{ACKNOWLEDGEMENTS}

This work has been funded by the Spanish MCYT under the project TEC 2005-06836-C0201.

\section{REFERENCES}

[1] A.Ferretti et a., "Permanent Scatterers in SAR Interferometry," IEEE TGRS, vol. 39, N.1, January 2001

[2] O.Mora, "Advanced Differential Interferometric SAR techniques for detection of terrain and building displacements," $\mathrm{PhD}$ Dissertation, UPC, Barcelona, March, 2004.

[3] L.Pipia et al., "Mining Induced Subsidence Monitoring in Urban Areas with a Ground-Based SAR," 2007 Urban Remote Sensing Joint Event, 11-13 April 2007,Paris.

[4] F.T.Ulaby,C.Elachi, "Radar Polarimetry for Geoscience Applications", Artech House Inc., 1990.

[5] L.Pipia et al., "Atmospheric Artifact Estimation and Compensation in Differential Polarimetric GB-SAR Acquisitions," EUSAR2006, Dresde, Germany 